Research Paper

\title{
Effects for Sequential Treatment of siAkt and Paclitaxel on Gastric Cancer Cell Lines
}

\author{
Minhee Ku 1,2, Myounghwa Kang 1, Jin-Suck Suh 1,2,3,4, Jaemoon Yang 1,3凶 \\ 1. Department of Radiology, College of Medicine, Yonsei University, Seoul 03722, Republic of Korea; \\ 2. Brain Korea 21 Plus Project for Medical Science, College of Medicine, Yonsei University, Seoul 03722, Republic of Korea; \\ 3. YUHS-KRIBB Medical Convergence Research Institute, Seoul 03722, Republic of Korea; \\ 4. Severance Biomedical Science Institute (SBSI), Seoul 03722, Republic of Korea \\ $\triangle$ Corresponding author: Jaemoon Yang, Assistant Professor, Systems Molecular Sensing Lab. Avison Bio-Medical Research Center (ABMRC), 50-1 Yonsei-ro, \\ Seodaemun-gu, Seoul, 03722, Republic of Korea. telephone +82 222280789 Fax +82222280376 email 177hum@yuhs.ac.
}

(C) Ivyspring International Publisher. Reproduction is permitted for personal, noncommercial use, provided that the article is in whole, unmodified, and properly cited. See http://ivyspring.com/terms for terms and conditions.

Received: 2016.03.10; Accepted: 2016.07.27; Published: 2016.09.07

\begin{abstract}
Real-time screening of cellular response on the drugs could provide valuable insights for the early detection of therapeutic efficiency and the evaluation of disease progression. Cancer cells have the ability to vary widely in response to stress in a manner to adjust the signaling pathway to promote the survival or having a resistance to stimulation. Cell-based label-free technologies using electronic impedance sensor have strategies for constructing the signature profiles of each cells. To achieve exquisite sensitivity to substantially change of live-cell response have an important role that predict the potential of therapeutic effects. In this study, we use an impedance-based real-time cell analysis system to investigate dynamic phenotypes of cells described as a cellular index value. We show that gastric cancer cells generated characteristic kinetic patterns that corresponded to the treatment order of therapeutics. The kinetic feature of the cells offers insightful information that cannot be acquired from a conventional single end-point assay. Furthermore, we employ a 'sequential treatment strategy' to increase cytotoxic effects with minimizing the use of chemotherapeutics. Specifically, treatment of paclitaxel (PTX) after down-regulating Akt gene expression using RNAi reduces the cell proliferation and increases apoptosis. We propose that the sequential treatment may exhibit more effective approach rather than traditional combination therapy. Moreover, the dynamic monitoring of cell-drug interaction enables us to obtain a better understanding of the temporal effects in vitro.
\end{abstract}

Key words: Akt; gastric cancer; paclitaxel (PTX); real-time cell analysis (RTCA); sequential treatment, small interfering RNA (siRNA).

\section{Introduction}

Paclitaxel (PTX), a microtubule-targeted drug, is one of the most widely used chemotherapeutic agents against ovarian, breast, brain and prostate cancers [1]. Recently, PTX has been tested in advanced gastric cancers and is now considered a key drug for clinical study $[2,3]$. PTX has been proven to block the growth and proliferation of cancer cells by preventing the disassembly and stabilizing of microtubules against depolymerization [4,5]. PTX induces cell death by apoptosis and regulates the expression of tumor suppressor genes and cytokines [6, 7]. However, PTX chemotherapy often results in serious chemo-resistance to PTX and its DNA-damaging effects [8]. Mechanistically, PTX is associated with elevated level of Akt that closely related to multiple cellular processes such as cell growth, proliferation, and cell migration $[9,10]$. To enhance the efficacy of cancer chemotherapy, various therapeutic strategies have been reported such as using combinations of signaling inhibitors, incorporation of adjuvant chemotherapy, down-regulation of apoptotic gene expression and thermo-chemotherapy [11, 12]. As with many anticancer drugs, the chemo-sensitivity of cancer cells must be increased in an effort to increase 
the effectiveness of PTX, otherwise, the usage of PTX must be minimized to reduce side effects; this may result in sub-therapeutic levels of drug. Targeting gene signaling pathways to improve therapeutic response is considered a suitable approach to these issues [13-15]. The chemo-sensitivity of cancer cells to PTX depends on the activation of a signal transduction pathway involved in cell proliferation [16]. Previous researches have suggested that the serine/threonine kinase Akt plays a prominent role as a key mediator of cellular survival pathways and contributes to chemotherapeutic resistance [17, 18]. Akt expression inhibits apoptosis through anti-apoptotic Bcl-2 family members and controls multiple intracellular targets. In addition, Akt regulates glycolytic activity that coordinately affects the cellular response to chemotherapeutic agents against selected critical targets of signaling pathways [18-21].

On the other hand, dysregulated cell metabolism has been linked to clinical relevant area for cancer therapy [22]. Cancer cells can be reprogrammed in bioenergetics and biosynthetic metabolism that results from multiple genetic changes and cellular abnormalities [23-25]. To predict the response of the cancer cells to therapeutic agents is convoluted argument because molecular mechanism of cancer cells is complicated and diverges significantly from those of normal cells. Therefore, in terms of the complexity of cancer progression mechanisms and heterogeneity, a significant problem for cancer therapy is how to overcome the anticancer drug resistance and how to detect and observe a change in the cellular response. Most of the usual approaches to monitor cellular responses after drug treatment only show a dose-dependent cytotoxic effects and the conclusion regarding the mechanism of action for the drug that has multiple and kinetically distinct effect based upon the time point. Our aim was to find effective strategy for cancer therapy by controlling variable condition such as the time point, dose concentration and order of sequential administration to elevate combination effects of the same drugs in cancer cells [26]. Moreover, it requires multiple variables to determine the changed molecular signaling pathways involved in tumor progression followed by balloon effects took place in response to an external stimulus. Therefore, we presented importance in combination and sequential treatment to elevate combination effects in cancer cells. For the evaluation of biological response to drug interaction with cells in entire course, in recent, the real-time cell analysis (RTCA) was used to quantitatively monitor the changes in cells during the course of our experiments [27]. RTCA measures the electrical impedance-based signals of adherent cells taken from an electronic sensor plate reflect changes in cellular parameters. The cellular index digitally represents cell proliferation, changes in adhesion and/or attachment of cells to microelectrode and cell morphology. RTCA is a novel tool that allows for label-free detection and long-term assay of live cells. Moreover, RTCA has a wide range of applications such as monitoring of cell-mediated cytotoxicity, screening of RNAi (RNA interference) effects and invasion/migration of cells [28]. These results provide evidence for the systemized therapeutic strategy should be developed to enhance the effectiveness of chemo-treatment without unwanted side effects and the real-time monitoring of cellular responses will be helpful to establish a more effective treatment strategy

In this study, the in-situ profiles for a proliferation of gastric cancer cells after RNAi and chemo-treatment in a sequential manner were monitored by RTCA. Here, small interfering RNA (siAkt) was used to specifically silence Akt oncogene expression and PTX was selected to disturb the stability of microtubules. The inhibition of Akt would extensively increase the PTX-induced cytotoxicity in gastric cancer cell lines. To predict the efficacy from the sequential treatment using siAkt and PTX, moreover, the treatment intervals and the order of therapeutic agents were controlled.

\section{Materials and methods}

\section{Cell culture}

Human gastric cancer cell lines (MKN28 and MKN45 cells) were obtained from the American Type Culture Collection (Manassas, VA, USA) and cultured at $37^{\circ} \mathrm{C}$ in $5 \% \mathrm{CO}_{2}$ humidified atmosphere in RPMI 1640 medium supplemented with $10 \%$ fetal bovine serum. Cellular morphology was observed using an Olympus ${ }^{\circledR}$ microscope and microscopic images were captured with an Olympus ${ }^{\circledR}$ digital camera.

\section{PTX treatment}

PTX was provided by Sigma-Aldrich (St. Louis, MO, USA, Cat. \#T7191) and dissolved in dimethyl sulfoxide (DMSO) as a $10 \mathrm{mM}$ stock solution. MKN28 and MKN5 cells were plate at $1 \times 10^{4}$ cells per well in 96-well plate. After incubating for $24 \mathrm{~h}$ at $37^{\circ} \mathrm{C}$, cells were incubated with PTX.

\section{siRNA transfection}

MKN28 and MKN45 cells were plated at $2 \times 10^{5}$ cells per well in 6 -well dishes and $1 \times 10^{4}$ cells per well in E-plate 16 to $70-80 \%$ confluence and transfected using Lipofectamine 2000 transfection reagent according to the manufacturer's protocol (Life Technologies, Inc., Gaithersburg, MD, USA). MKN28 
and MKN45 cells were transfected with the siRNA for knockdown of Akt (ON-TARGETplus Human Akt1 (207) siRNA-SMARTpool, Cat. \#L-003000-00-0010, Dharmacon, Lafayette, CO, USA), and scrambled siRNA (ON-TARGETplus Non-targeting pool, Cat. \#D-001810-10, Dharmacon) at $100 \mathrm{nM}$ final concentration using Lipofectamine 2000 and Opti-MEM medium following the protocols recommended by the manufacturer (Thermo Scientific, Waltham, MA, USA).

\section{Real-time Cell Analysis (RTCA)}

Real-time cellular proliferations for MKN28 and MKN45 cells were analyzed using the $x$ CELLigence ${ }^{\mathrm{TM}}$ DP system (Roche Diagnostics GmbH, Berlin, Germany). For the monitoring of cell index, MKN28 and MKN45 cells were seeded in the E-plate 16 (ACEA Biosciences, San Diego, CA, USA) at a density of $1 \times 10^{4}$ cells per well and incubated for $24 \mathrm{~h}$. After $24 \mathrm{~h}$, the cells were tested using five experimental conditions: DMSO-treated cells as a control (NT, •), siAkt transfection (siAkt only, $\circ$ ), simultaneous treatment of siAkt and PTX (siAkt \& PTX, $\mathbf{\nabla}$ ), siAkt transfection after PTX treatment in sequential manner $($ PTX $\rightarrow$ siAkt, $\Delta)$ and PTX treatment after siAkt transfection in sequential manner (siAkt $\rightarrow$ PTX, $\mathbf{~})$. According to these treatment conditions, the cells were incubated at $37^{\circ} \mathrm{C}$ in a $5 \% \mathrm{CO}_{2}$ humidified atmosphere and automatically monitored real-time at every $1 \mathrm{~h}$ by the xCELLigence system and expressed as a CI (cell index) value. The CI calculation is based on the following formula: $\mathrm{CI}=\left(\mathrm{Z}_{\mathrm{i}}-\mathrm{Z}_{0}\right) / 15_{S}\left(\mathrm{Z}_{\mathrm{i}}\right.$ : the impedance at an individual point of time during the experiment, $Z_{0}$ : the impedance at the start of the experiment) [29]. Data for cell adherence were normalized at $24 \mathrm{~h}$ after cell seeding. Normalized CI is calculated by dividing $\mathrm{CI}$ at the normalized time into the original CI. All experiments were performed in triplicate and the average and standard deviation were reported.

\section{Quantitative real-time PCR}

Total RNA was extracted from harvested gastric cancer cells using the Ambion mirVana ${ }^{\mathrm{TM}}$ miRNA Isolation Kit (Cat \# AM1560, Ambion, Austin, TX, USA). The quality of the isolated RNA was assessed using a NanoDrop Lite Spectrophotometer (Thermo Scientific). All samples had a 260/280 ratio of $\sim 2.0$. Total RNA was converted to cDNA using the high capacity RNA-to-cDNA kit (Cat \# 4387406, Applied Biosystems, Carlsbad, CA, USA) according to the manufacturer's recommendation. cDNA synthesis using $1 \mu \mathrm{g}$ of RNA per $20 \mu \mathrm{L}$ reaction was performed using the Roche LightCycler ${ }^{\circledR}$ system (Roche Diagnostics). Quantitative real-time PCR was performed in triplicate using HiFast SYBR Lo-Rox reagents (Cat. \#Q100240, GenePool, Edinburgh, UK). Thermo-cycling conditions were as follows: initial denaturation at $95^{\circ} \mathrm{C}$ for $10 \mathrm{~min}$ followed by 45 cycles at $95^{\circ} \mathrm{C}$ for $10 \mathrm{sec}$ and $60^{\circ} \mathrm{C}$ for $30 \mathrm{sec}$ (annealing and extension). Sequences of specific primer sets used in this study are listed in Table 1. Primer sequences were designed using the Primer3 software (http://frodo.wi.mit.edu/primer3/). The 2- $\Delta \Delta \mathrm{Ct}$ method was used to calculate fold differences in gene expression, using the beta-Actin gene ( $\beta$-actin) as housekeeping reference for data normalization. PCR products were subjected to melting curve analysis to rule out the synthesis of non-specific products.

Table 1. mRNA primer sequences used for Quantitative real-time PCR analysis.

\begin{tabular}{|c|c|}
\hline Target Gene & Primer Sequence \\
\hline AKT & $\begin{array}{l}\text { Forward: TCT ATG GCG CTG AGA TTG TG } \\
\text { Reverse: CTT AAT GTG CCC GTC CTT GT }\end{array}$ \\
\hline Bcl-xL & $\begin{array}{l}\text { Forward: GCG TGG AAA GCG TAG ACA AG } \\
\text { Reverse: TGC TGC ATT GTT CCC ATA GA }\end{array}$ \\
\hline Bcl-2 & $\begin{array}{l}\text { Forward: GTT GCT TTA CGT GGC CTG TT } \\
\text { Reverse: CAG GTT TCC TGC TTT CTT GG }\end{array}$ \\
\hline Bad & $\begin{array}{l}\text { Forward: GCC GAG TGA GCA GGA AGA } \\
\text { Reverse: ACT GGC GTC CCA CAG GAG }\end{array}$ \\
\hline Caspase3 & $\begin{array}{l}\text { Forward: AAG ATC ACA GCA AAA GGA GCA } \\
\text { Reverse: CAA CGA TCC CCT CTG AAA AA }\end{array}$ \\
\hline$\beta$-actin & $\begin{array}{l}\text { Forward: CTC TTC CAG CCT TCC TTC CT } \\
\text { Reverse: TGT TGG CGT ACA GGT CTT TG }\end{array}$ \\
\hline
\end{tabular}

\section{Statistical analysis}

In vitro results are expressed as mean \pm standard deviation. Student's t-test was performed to determine statistically significant differences between groups, and a $\mathrm{p}$ values $(<0.01$ or 0.05$)$ were considered statistically significant. Error bars denote the standard error $(n=3)$.

\section{Results}

\section{Chemo-sensitivity of PTX to gastric cancer cell lines}

To investigate the chemo-cytotoxic effect of PTX on gastric cancer cell lines, cellular proliferation was monitored by a real-time and label-free method. The microelectrodes measure the electrical impedance that reflects interaction between cell population and the sensor surface in each well and it provides quantitative kinetic trace about the status of the cells [30]. MKN28 and MKN45 cells were respectively seeded into microelectrodes-deposited wells and treated with PTX at various concentrations from $10^{\circ}$ to $10^{4} \mathrm{nM}$. At higher PTX concentrations, round cellular morphology was observed in both PTX-treated MKN28 and MKN45 cells compared to the flat control 
cells (Figure 1A). The cytotoxic effects of PTX on both MKN28 and MKN45 cells were subsequently monitored for $24 \mathrm{~h}$ after the treatment of PTX. Compared to DMSO control without PTX treatment, the normalized cell indexes for MKN28 and MKN45 cells presented remarkable differences as an increase of PTX concentration (Figure 1B). The $50 \%$ inhibitory concentration $\left(\mathrm{IC}_{50}\right)$ values of PTX for MKN28 (23 nM) or MKN45 cells $(60 \mathrm{nM})$ were calculated after $24 \mathrm{~h}$ exposure to PTX (Figure 1C).

\section{Specific modulation of Akt expression in gastric cancer cell lines}

In order to determine the optimal transfection condition of siRNA, gel retardation assay was performed to evaluate the degree of binding between Lipofectamine and siAkt at varying weight ratios. A series of Lipofectamine/siAkt complexes in ratios $(\mathrm{w} / \mathrm{w})$ of $0.25: 1,0.5: 1,1: 1,2: 1$, and $4: 1$ were examined and 1:1 ratio was selected for the target gene down-regulation assay. Thus, MKN28 and MKN45 cells were respectively transfected with 50,100, and $200 \mathrm{nM}$ of siAkt and $50 \mathrm{nM}$ scrambled siRNA (siScr) as a control to evaluate the transfection efficiency of siAkt. Clear down-regulations of Akt mRNA for both cell lines were revealed by quantitative real-time PCR (Figure 2A and 2B). The inhibitory effect in gene regulation was minimal at $50 \mathrm{nM}$ of siAkt in both
MKN28 and MKN45 cells. In contrast, over $100 \mathrm{nM}$ of siAkt exhibited effective inhibitory effect in Akt mRNA expression for both MKN28 and MKN45 cells rather than the other transfection conditions. Subsequently, normalized cell indexes for cellular proliferations of MKN28 and MKN45 cells were monitored (Figure 2C and 2D). In both cell lines, the reduced proliferations were remarkably observed at 100 and $200 \mathrm{nM}$ of siAkt $(\mathrm{p}<0.01)$.

\section{Sequential treatment of siAkt and PTX for MKN28 cells}

To investigate the cytotoxic efficacy of sequential treatment of siAkt and PTX, the proliferation for two gastric cancer cells were monitored under five different treatment conditions (Figure 3A); non-treatment control (NT $\rightarrow \mathrm{NT}, \mathrm{CTRL}, \bullet)$, siAkt transfection (siAkt $\rightarrow \mathrm{NT}$, siAkt only, o), simultaneous treatment of siAkt and PTX (siAkt \& PTX $\rightarrow \mathrm{NT}, \boldsymbol{\nabla}$ ), siAkt transfection after PTX treatment in sequential steps (PTX $\rightarrow$ siAkt, $\Delta$ ), and PTX treatment after siAkt transfection in sequential steps (siAkt $\rightarrow$ PTX, $\mathbf{})$. Here, concentrations of siAkt and PTX were $100 \mathrm{nM}$ and 70 $\mathrm{nM}$ to evaluate the cytotoxic efficacy from the sequential treatment, respectively. In order to determine the proper interval time point of the sequential treatment, time intervals between the first and second treatment were $12 \mathrm{~h}, 24 \mathrm{~h}$, and $48 \mathrm{~h}$.
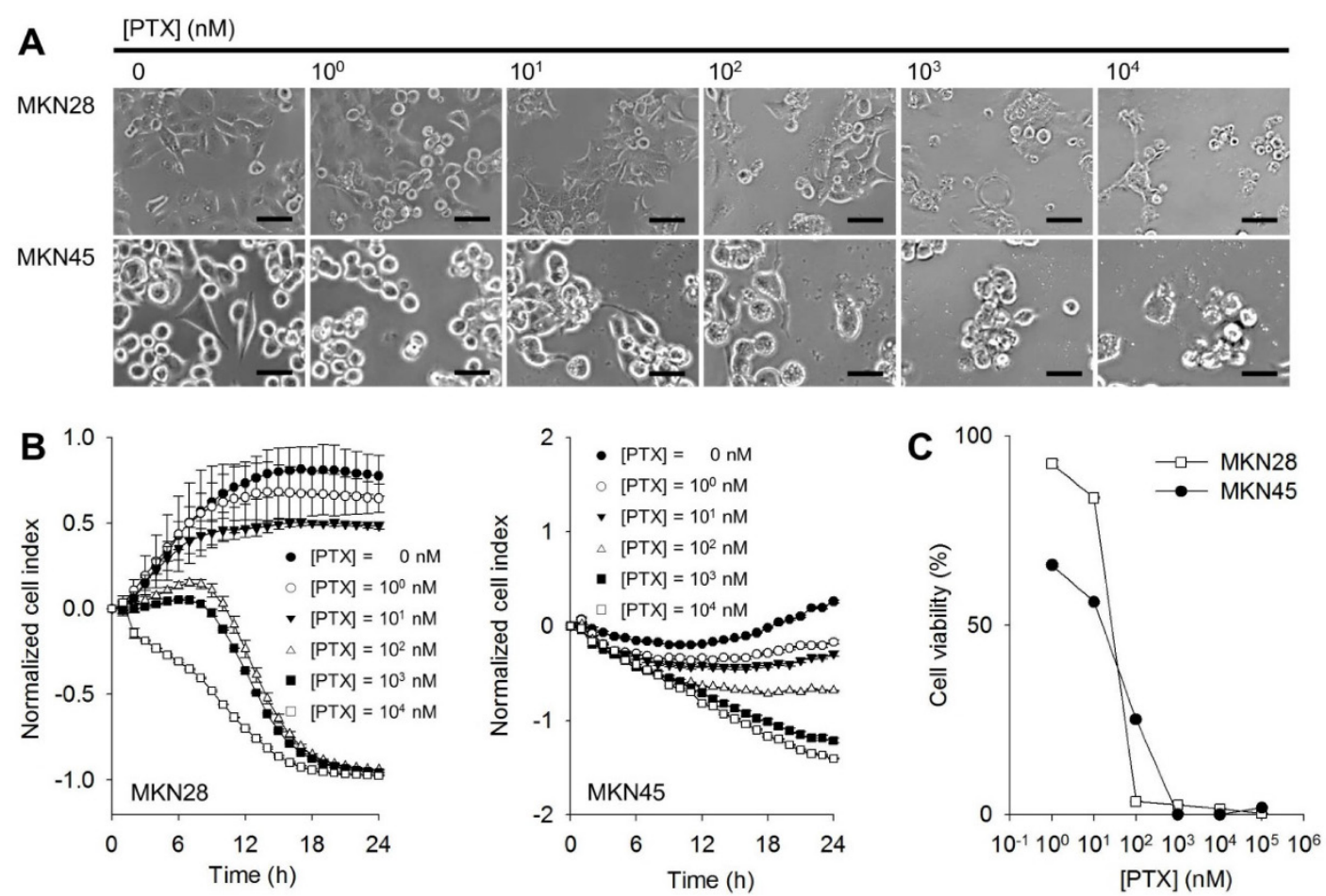

Figure 1. Chemo-sensitivity of PTX on gastric cancer cell lines. (A) Cellular microscopic images for MKN28 and MKN45 cells at $24 \mathrm{~h}$ after addition of PTX. Scale bars mean $50 \mu \mathrm{m}$. (B) Proliferation profiles for MKN28 (left) and MKN45 (right) cells obtained by the RTCA after $24 \mathrm{~h}$ from PTX treatments (0 - $10^{4} \mathrm{nM}$, 1:10 serial dilutions). All graphs represent three independent experiments and with standard deviations $(n=3)$. (C) Cell viabilities for MKN28 and MKN45 cells calculated from $(B)$ at $24 \mathrm{~h}$ from PTX treatment. 
Morphological change and rounding up in the cells due to the cellular damage from sequential treatment were observed (Figure 3B). At both $24 \mathrm{~h}$ and $48 \mathrm{~h}$ of time intervals, siAkt $\rightarrow$ PTX condition $(\square)$ exhibited the greatest cytotoxic efficacy compared to other conditions (Figure. 3C). At $12 \mathrm{~h}$ of time interval, interestingly, both siAkt $\rightarrow \mathrm{NT} \quad(\circ)$ and siAkt \& PTX $\rightarrow$ NT $(\boldsymbol{\nabla})$ conditions presented the rebound of cell proliferation from $12 \mathrm{~h}$ after the treatment of therapeutics. Only siAkt $\rightarrow$ PTX (a) condition appeared continuous cytotoxic effect during a monitoring period. Furthermore, there was no effective reduction of cell proliferation under only siAkt transfection condition (siAkt $\rightarrow \mathrm{NT}, \circ$ ). As shown in Figure. 3D, the case for $24 \mathrm{~h}$ of time interval exhibited the greatest cytotoxic effect with $63 \%$ rather than any other treatment groups.

\section{Sequential treatment of siAkt and PTX for MKN45 cells}

As indicated in Figure 4A, the cytotoxic efficacies for MKN45 cells were evaluated by five sequential treatment conditions. Similar to the case of MKN28 cells, cellular damages were observed by a microscopy after the sequential treatments (Figure 4B). However, the cytotoxic capacity from siAkt $\rightarrow$ PTX (a) condition using $100 \mathrm{nM}$ of siAkt was not significant and reduced normalized cell index was $53 \%$ (Figure $4 \mathrm{C}$ and $4 \mathrm{D}$ ). When the transfection

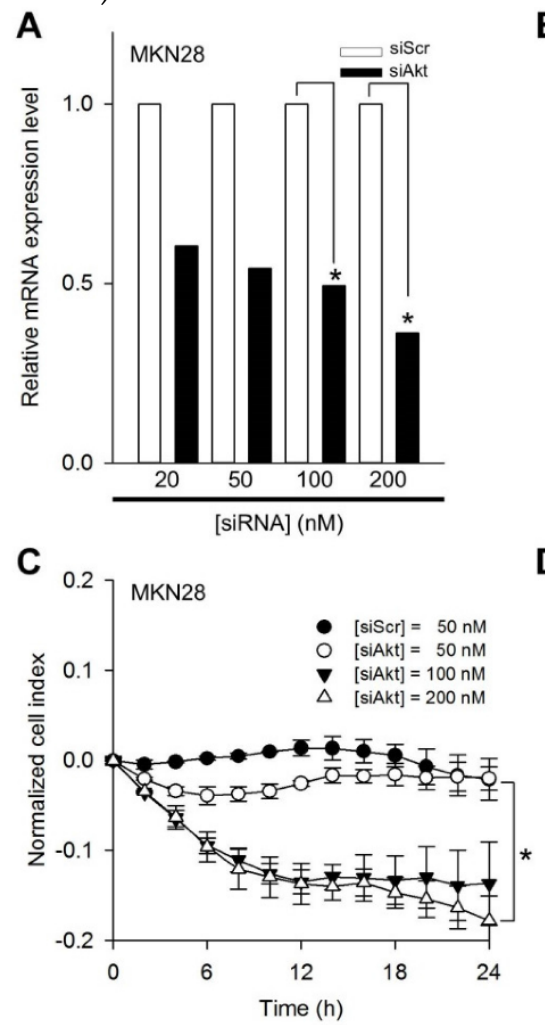

concentration of siAkt was increased with $200 \mathrm{nM}$, the enhancement of cytotoxic effect against MKN45 cells was confirmed after the sequential treatment of siAkt and PTX (Figure 4C). Normalized cell index for siAkt $\rightarrow$ PTX (ロ) condition was gradually decreased and $\Delta$ normalized cell index was lowest with $87 \%$ in MKN45 cells compared to other treatment conditions.

\section{Apoptotic effects of sequential treatment of siAkt and PTX}

To analyze apoptotic effects on MKN28 and MKN45 cells by the sequential treatment of siAkt and PTX, the expressions of apoptosis-related genes were investigated using quantitative real-time PCR. As shown Figure 5, the level of Akt expression for siAkt $\rightarrow$ PTX ( $(\mathbf{)})$ condition was significantly lower than $(\mathrm{NT} \rightarrow \mathrm{NT}, \mathrm{CTRL}, \bullet)$ in both MKN28 and MKN45 cells. On the contrary, we found that Akt gene expression was elevated when siAkt was used after PTX treatment. Furthermore, we focused on the expression levels of pro-apoptotic Caspase- 3 and Bad proteins and anti-apoptotic $\mathrm{Bcl}-\mathrm{xL}$ and $\mathrm{Bcl}-2$ proteins. The sequential treatment of siAkt and PTX induces molecular events that increase pro-apoptotic proteins and decrease anti-apoptotic proteins, relatively. The mRNA expression levels of Bcl-xL and Bcl-2 were decreased in both MKN28 and MKN45 cells, whereas the mRNA expression levels of Caspase- 3 tended to be inconsistent.

B

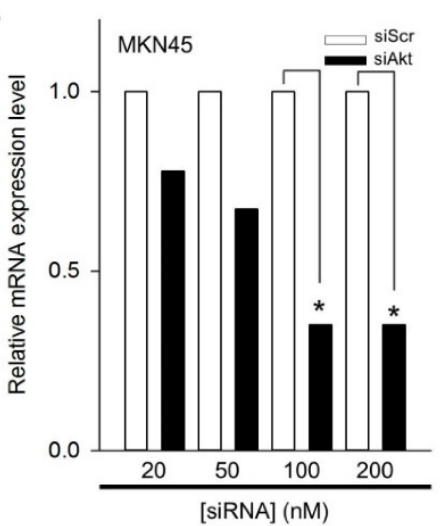

D

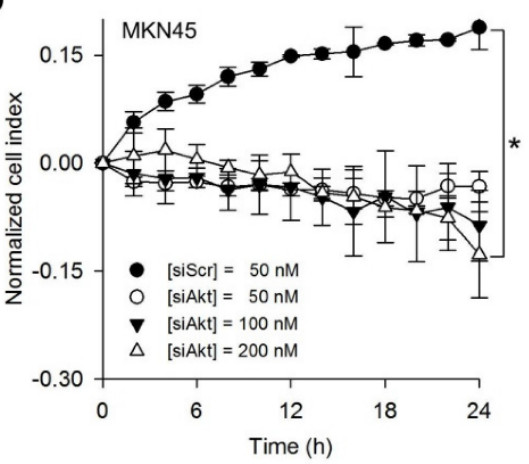

Figure 2. Effects of siAkt on gastric cancer cell lines. Relative Akt mRNA expression levels as a function of siScr and siAkt in a dose-dependent manner for (A) MKN28 and (B) MKN45 cells. Proliferation profiles monitored by RTCA for (C) MKN28 and (D) MKN45 cells treated with $50 \mathrm{nM}$ of scrambled siRNA (siScr) and 50, 100, and 200 nM of siAkt, respectively. The normalized cell index was calculated every $2 \mathrm{~h}$. All graphs represent three independent experiments and with standard deviations $(n=3)$. ${ }^{p}<0.01$. 

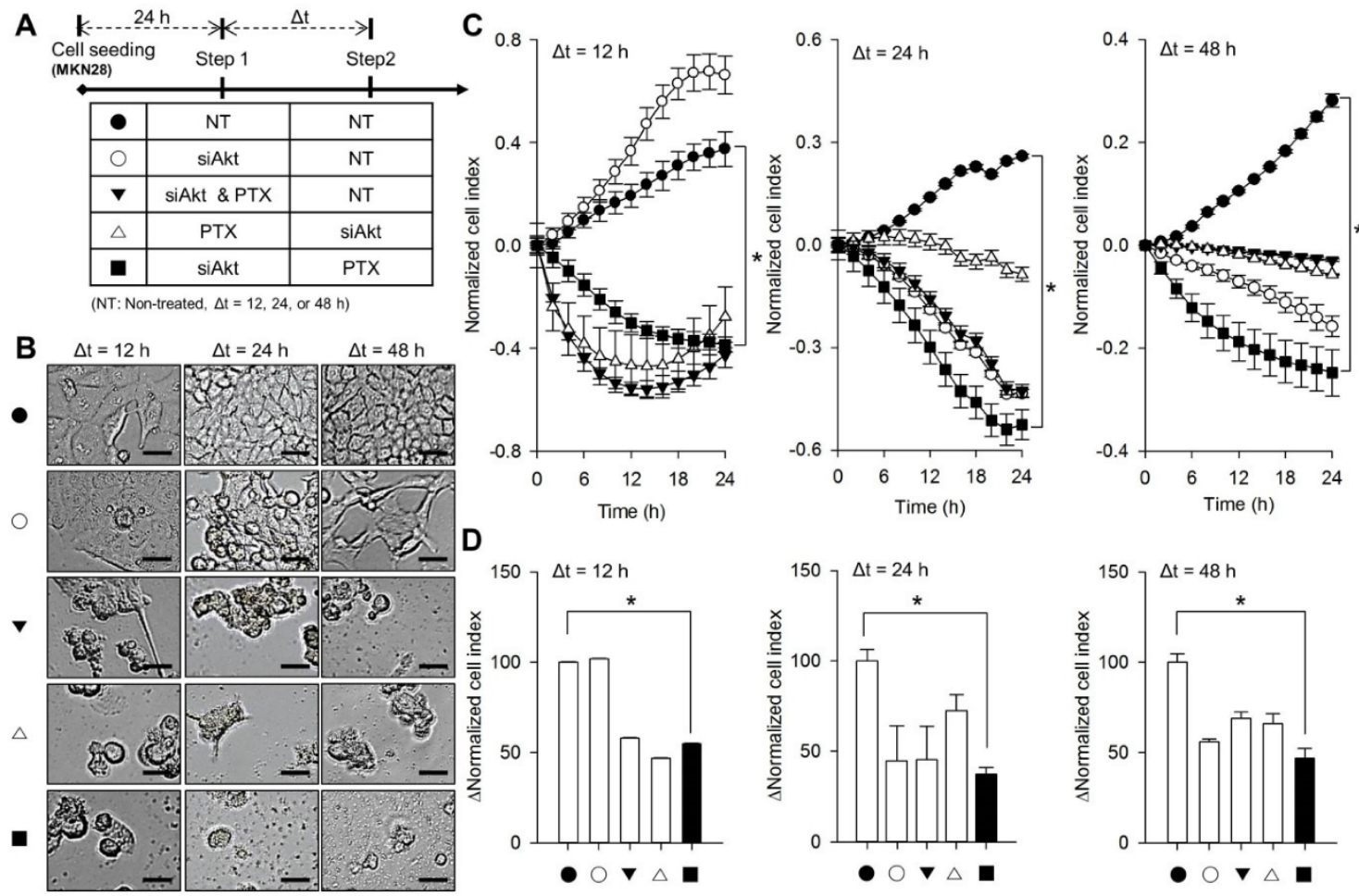

Figure 3. Sequential treatment of siAkt and PTX against MKN28 cells. (A) The index for sequential treatment of siAkt and PTX; DMSO treatment as a control (NT, $\bullet$ ), siAkt transfection (siAkt only, o), simultaneous treatment of siAkt and PTX (siAkt \& PTX, $\mathbf{\nabla}$ ), siAkt transfection after PTX treatment in sequential manner (PTX $\rightarrow$ siAkt, $\Delta$ ), and PTX treatment after siAkt transfection in sequential manner (siAkt $\rightarrow$ PTX, $\mathbf{a})$. (B) Cellular microscopic images for MKN28 cells after the sequential treatment of siAkt and PTX at the indicated treatment time; $\Delta t=12,24$, and $48 \mathrm{~h}$. (C) Normalized proliferation profiles of MKN28 cells after the sequential treatment of siAkt and PTX. (D) $\Delta$ Normalized cell index calculated from (C). Non-treatment condition was used as a control. ${ }^{*} \mathrm{p}<0.01$.
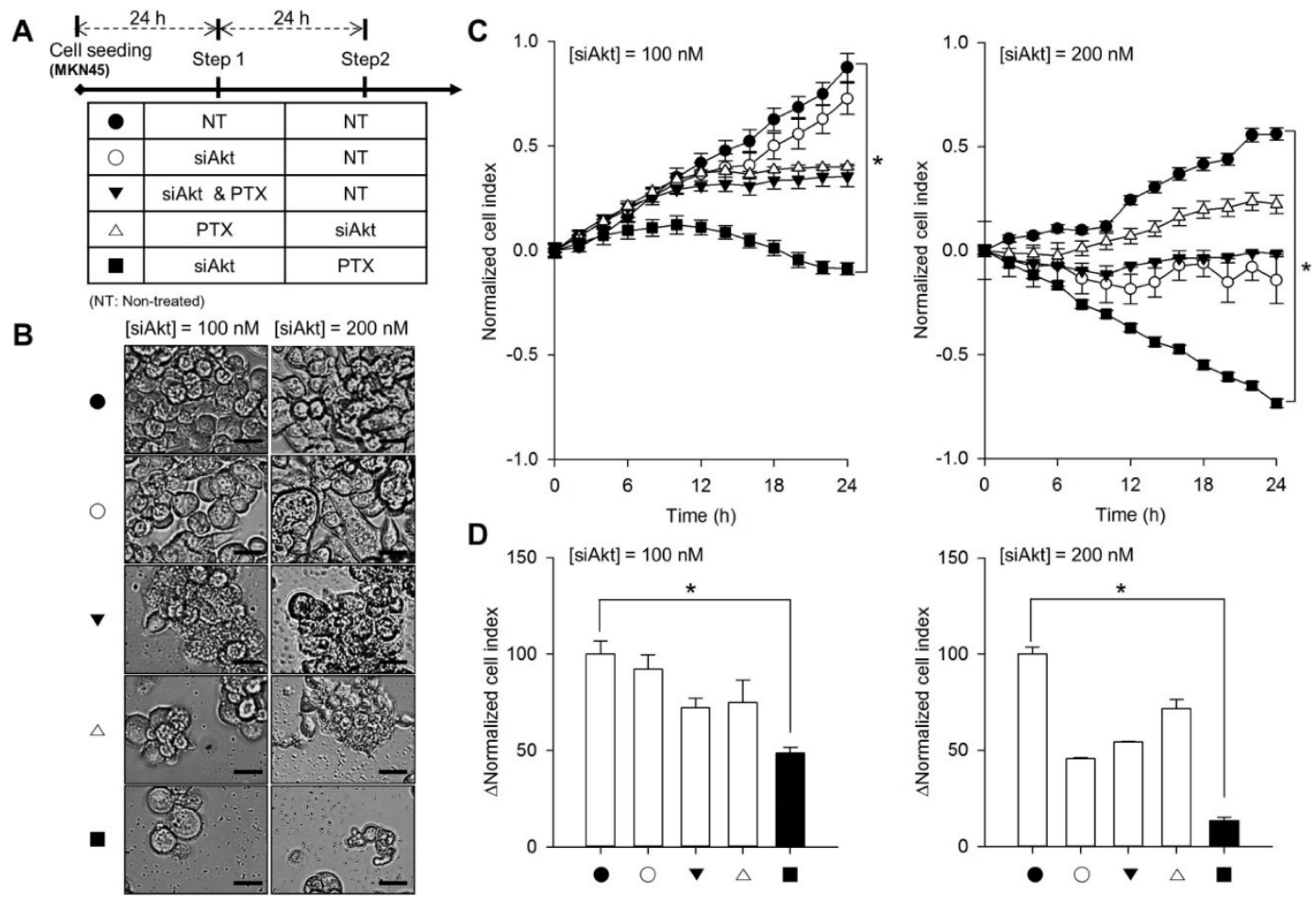

Figure 4. Sequential treatment of siAkt and PTX against MKN45 cells. (A) The index for sequential treatment of siAkt and PTX; DMSO treatment as a control (NT, $\bullet$ ), siAkt transfection (siAkt only, o), simultaneous treatment of siAkt and PTX (siAkt \& PTX, $\mathbf{\nabla}$ ), siAkt transfection after PTX treatment in sequential manner (PTX $\rightarrow$ siAkt, $\Delta$ ), and PTX treatment after siAkt transfection in sequential manner (siAkt $\rightarrow$ PTX, - ). (B) Cellular microscopic images for MKN45 cells after the sequential treatment of siAkt and PTX at $24 \mathrm{~h}$ of treatment time; 100 (left column) and $200 \mathrm{nM}$ (right column) of siAkt. (C) Normalized proliferation profiles of MKN45 cells after the sequential treatment of siAkt and PTX; 100 (left) and $200 \mathrm{nM}$ (right) of siAkt. (D) $\Delta$ Normalized cell index calculated from (C). Non-treatment condition was used as a control. * ${ }_{\mathrm{p}}<0.01$. 


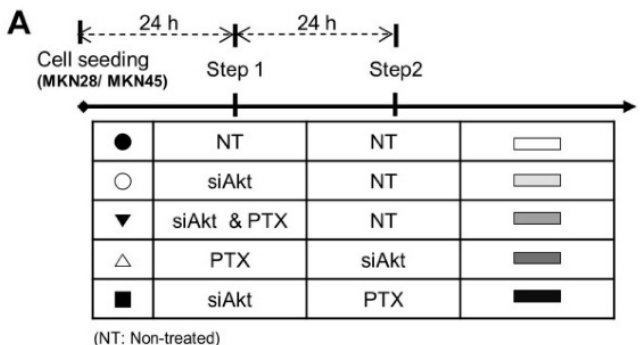

B

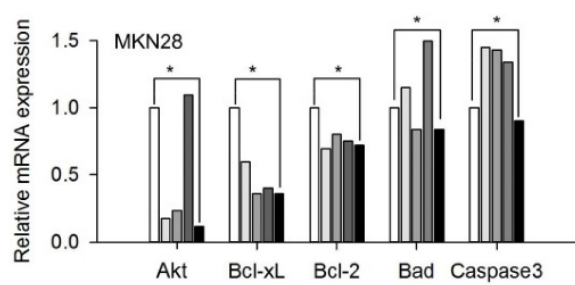

C

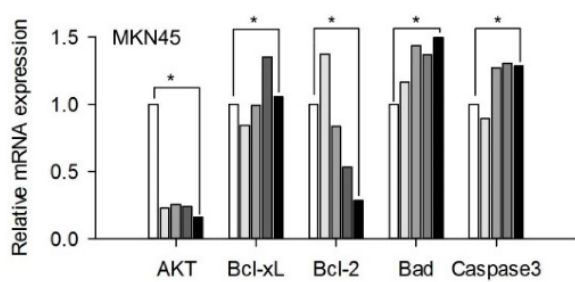

Figure 5. Apoptosis-related gene expressions after the sequential treatment of siAkt and PTX on gastric cancer cell lines. (A) The index for sequential treatment of siAkt and PTX; DMSO treatment as a control (NT, •), siAkt transfection (siAkt only, o), simultaneous treatment of siAkt and PTX (siAkt \& PTX, $\boldsymbol{\nabla})$, siAkt transfection after PTX treatment in sequential manner (PTX $\rightarrow$ siAkt, $\Delta$ ), and PTX treatment after siAkt transfection in sequential manner (siAkt $\rightarrow$ PTX, $\mathbf{\square})$. Total RNA was extracted from the cells at $24 \mathrm{~h}$ of treatment time. The RNA samples were subjected to qRT-PCR to analyze apoptosis-associated genes (Akt. Bcl-xL, Bcl-2, Bad, and Caspase-3) of interest in (B) MKN28 and (C) MKN45 cells. ${ }^{*} \mathrm{p}<0.01$.

\section{Discussion}

PTX, the anti-mitotic antitumor drug, has been extensively used for the treatment of a variety of human gastric cancers [31]. PTX suppresses microtubule dynamics and function via control of cell signaling, mitotic activity and proliferative capacity [4]. Microtubules are the major cytoskeletal components and are considered as an essential target for anticancer therapy [5]. Although microtubule-targeted PTX has shown clinical success, drug resistance and cellular toxicity frequently lead to cancer treatment failure [7, 32]. In particular, cancer cells develop complex mechanisms to evade the chemotherapeutic effect of the drug. These molecular mechanisms induce drug resistance as well as lower sensitivity to drugs [33]. To decrease chemotherapeutic resistance and increase the effectiveness of chemotherapy for refractory cancers, a strategy using combination therapy targeting apoptosis-related pathways still represents one of the best solutions.

On thither hand, Akt plays a major role in a fundamental signaling pathway that includes cell proliferation, growth and survival. Akt has previously been demonstrated as a key protein associated with chemo-sensitivity for a variety of cancers [17]. In addition, Akt-related signaling pathway can control glucose metabolism and cellular energy mechanism and acts as a positive regulator of numerous downstream targets to shift the apoptotic threshold in cancer cells [34-36]. Moreover, Akt signaling inactivates pro-apoptotic factors, including the Bcl-2 family, and caspase-9, and also up-regulates anti-apoptotic genes by activating transcription factors [34]. Therefore, Akt is a reasonable target for the development of chemotherapeutics against a central node in the cell survival signaling process.

As discussed, our study tested a regimen combining standard chemotherapy and RNAi to block signaling pathways that may influence chemo-sensitivity in gastric cancer. In particular, the combination treatment of chemotherapeutics and RNAi has been conducted in sequential manner. RNAi-mediated knockdown of specific gene has been confirmed as an effective method to inhibit target protein expression [37]. Short interfering RNA (siRNA) has potential as a more intelligent therapeutic approach by specifically and efficiently recognizing a single point in targeted gene expression [38]. Thus, RNAi has been considered a potent chemo-enhancer to existing chemotherapy agents [39]. In this study, we investigated whether Akt down-regulation would result in increased chemotherapeutic efficacy of PTX in human gastric cancer cell lines. By comparing dosing interval and exposure order of siAkt and PTX, we found that the sequential treatment was more effective when combining two therapeutic drugs that act respectively via different mechanisms. Hence, we focused on sequential treatment using Akt-regulating siRNA using a conventional transfection reagent and the anticancer drug PTX on two gastric cancer cell lines. We have conducted a series of in vitro studies under various treatment conditions such as non-treatment as a control, siAkt transfection, siAkt and PTX simultaneously treatment, siAkt transfection after PTX-treatment in a sequential manner and PTX-treatment after siAkt transfection in a sequential manner to compare the cytotoxic effects. We used two gastric cancer cell lines (MKN28 and MKN45) to investigate changes in gene expressions and cytotoxicity from the sequential treatment of siAkt and PTX.

On the other hand, in vitro assessment of the cell viability and proliferation pattern of cancer cells is essential for the understanding of cytotoxic effects and a screening of meaningful therapeutics. However, the conventional tetrazolium-based viability assays reveal the endpoint results that can ignore the 
relevant in-situ biochemical event in living cells. To obtain the data for cellular viabilities at numerous time points, moreover, abundant cell samples are needed. To address these limitations, in recent, the impedance-based real-time cell analyzing system has developed and extended to a wide range of in vitro applications for label-free detection of cell processes directly [40]. Here, the impedance value demonstrates the collected information of cellular events that include the relative density, changes in cell morphology, attachment and spreading of cell on the electrodes that occur over several days. Therefore, the extended validation by a dynamic monitoring of cellular plasticity and the progression of cancer cells is significant to test the overall efficiency of therapeutics.

A major finding from our experiments was that when cells were pretreated with $100 \mathrm{nM}$ of siAkt for $24 \mathrm{~h}$ before incubation with a low dose of PTX, at lower concentration than approximating the $\mathrm{IC}_{50}$ of PTX in both two cell lines, the cytotoxic effects were enhanced. Our data present that PTX may drive the elevation of Akt level, thereby promoting survival and resistance to RNAi treatment. In contrast, inhibition of Akt expression via siRNA led to increased PTX-induced cytotoxic effect against both MKN28 and MKN45 cells (Figure. 5F). Therefore, our results also raise questions about the importance of the sequence-dependent regimen in the clinical formulation of the multi-target therapy. In particular, there are no critical differences in cell indexes and mRNA levels of Akt after the transfection of siAkt with 100 and $200 \mathrm{nM}$. When PTX was treated in siAkt-transfected gastric cancer cells, however, distinct changes of cell index were observed at each siAkt transfection condition. Various previous studies have reported that the regulation of Akt-related signaling might change a cellular fate and the sensitivity and/or resistance in treated therapeutics $[41,42]$. It means that the dose-dependent control of Akt signaling may affect PTX-sensitivity in gastric cancer cells. To better understand the mechanism of apoptosis by sequential treatment, we compared the expression levels of pro-/anti- apoptotic-related genes that are associated with multiple intracellular signaling pathways. The present results indicated that Akt has been shown to regulate cell survival and suppress apoptosis [21]. Therefore, we investigated Bcl-2 family proteins involved in the anti-apoptotic effects and Akt and caspase-3 expression, which interact to regulate programmed cell death $[43,44]$. Results showed that sequential treatment of PTX-treatment after siAkt-transfection slightly increased the expression levels of caspase-3 and dominantly decreased Bcl-xL and Bcl-2 gene levels. The results presented that the level of anti-apoptotic signal was significantly correlated with cancer cell apoptosis, support our concept of sequential therapy.

In conclusion, our findings demonstrated that the anticipative suppression of Akt expression and sequential PTX-treatment had increased the cytotoxic effect toward gastric cancer cell lines. In particular, the real-time profiling of the cellular proliferating state as a reaction to therapeutic compounds in sequential steps enable to clearly elucidate the optimal treatment step that influenced on multiple cells. The treatment of siAkt and PTX in a sequential manner has induced the apoptosis of gastric cancer cells compared to single treatment of siAkt or PTX. These findings provide a strong rationale for establishment of a promising strategy for clinical trials with anticipative suppression of Akt expression and sequential PTX-treatment on gastric cancer. In addition, our observations in gastric cancer cells are being expanded to undertake similar studies in a variety of human cancer cell lines. Further characterization of the sequential treatment for therapeutic efficiency of RNAi and chemotherapeutics will be helpful for clinical utility.

\section{Abbreviations}

BSA: bovine serum albumin; cDNA: DNA complementary to RNA; DMSO: dimethylsulfoxide; DNA: deoxyribonucleic acid; FBS: fetal bovine serum; GAPDH: glyceraldehyde-3-phosphate dehydrogenase; IC50: concentration giving half-maximal inhibition; mRNA: messenger RNA; PBS: phosphate-buffered saline; PCR: polymerase chain reaction; RNA: ribonucleic acid; RNAi: RNA interference; PTX: Paclitaxel; SDS: Sodium dodecyl sulfate; siRNA: small interfering RNA; siAkt: AKT-targeted small interfering RNA.

\section{Acknowledgements}

This work was supported by the National Research Foundation of Korea (NRF) grant funded by the Korea government, Ministry of Education and Science Technology (MEST) (NRF-2014R1A1A205 9806).

\section{Competing Interests}

The authors have declared that no competing interest exists.

\section{References}

1. Rowinsky EK, Donehower RC. Paclitaxel (taxol). N Engl J Med. 1995; 332: 1004-14.

2. Zhou H-B, Zhu J-R. Paclitaxel induces apoptosis in human gastric carcinoma cells. World J Gastroenterol. 2003; 9: 442-5.

3. Kulig J, Kołodziejczyk P, Kulig P, Legutko J. Targeted therapy for gastric cancer - Current status. Journal of Oncology Pharmacy Practice. 2013; 19: 75-81.

4. Dumontet C, Jordan MA. Microtubule-binding agents: a dynamic field of cancer therapeutics. Nature reviews Drug discovery. 2010; 9: 790-803. 
5. Jordan MA, Wilson L. Microtubules as a target for anticancer drugs. Nat Rev Cancer. 2004; 4: 253-65.

6. Szanto A, Hellebrand EE, Bognar Z, Tucsek Z, Szabo A, Gallyas F, Jr., et al. PARP-1 inhibition-induced activation of PI-3-kinase-Akt pathway promotes resistance to taxol. Biochemical pharmacology. 2009; 77: 1348-57.

7. Gascoigne KE, Taylor SS. How do anti-mitotic drugs kill cancer cells? Journal of Cell Science. 2009; 122: 2579-85.

8. Mitchison TJ. The proliferation rate paradox in antimitotic chemotherapy. Molecular biology of the cell. 2012; 23: 1-6.

9. Bava SV, Puliappadamba VT, Deepti A, Nair A, Karunagaran D, Anto RJ. Sensitization of taxol-induced apoptosis by curcumin involves down-regulation of nuclear factor-kappaB and the serine/threonine kinase Akt and is independent of tubulin polymerization. The Journal of biological chemistry. 2005; 280: 6301-8.

10. Page C, Lin HJ, Jin Y, Castle VP, Nunez G, Wuang M, et al. Overexpression of Akt/AKT can modulate chemotherapy-induced apoptosis. Anticancer Res. 2000; 20: 407-16.

11. Ganesh S, Iyer AK, Weiler J, Morrissey DV, Amiji MM. Combination of siRNA-directed Gene Silencing With Cisplatin Reverses Drug Resistance in Human Non-small Cell Lung Cancer. Molecular therapy Nucleic acids. 2013; 2: e110.

12. Chen S, Liu X, Gong W, Yang H, Luo D, Zuo X, et al. Combination therapy with VEGFR2 and EGFR siRNA enhances the antitumor effect of cisplatin in non-small cell lung cancer xenografts. Oncology reports. 2013; 29: 260-8.

13. Nagaraj NS, Datta PK. Targeting the transforming growth factor-beta signaling pathway in human cancer. Expert opinion on investigational drugs. 2010; 19: 77-91.

14. Porta C, Paglino C, Mosca A. Targeting PI3K/Akt/mTOR Signaling in Cancer. Frontiers in oncology. 2014; 4: 64

15. Seshacharyulu P, Ponnusamy MP, Haridas D, Jain M, Ganti AK, Batra SK. Targeting the EGFR signaling pathway in cancer therapy. Expert opinion on therapeutic targets. 2012; 16: 15-31.

16. Sunters A, Madureira PA, Pomeranz KM, Aubert M, Brosens JJ, Cook SJ, et al. Paclitaxel-induced nuclear translocation of FOXO3a in breast cancer cells is mediated by c-Jun NH2-terminal kinase and Akt. Cancer research. 2006; 66: 212-20.

17. Clark AS, West K, Streicher S. Constitutive and Inducible Akt Activity Promotes Resistance to Chemotherapy, Trastuzumab, Tamoxifen. Mol Cancer Ther. 2002; 1: 707-17.

18. Wang H, Duan L, Zou Z, Li H, Yuan S, Chen X, et al. Activation of the $\mathrm{PI} 3 \mathrm{~K} / \mathrm{Akt} / \mathrm{mTOR} / \mathrm{p} 70 \mathrm{~S} 6 \mathrm{~K}$ pathway is involved in S100A4-induced viability and migration in colorectal cancer cells. International journal of medical sciences. 2014; 11: 841-9.

19. MacKeigan JP, Taxman DJ, Hunter D. Inactivation of the antiapoptotic phosphatidylinositol 3-kinase-Akt pathway by the combined treatment of taxol and mitogen-activated Protein Kinase Kinase Inhibition. Clin Cancer Res. 2002; 8: 2091-9.

20. VanderWeele DJ, Zhou R, Rudin CM. Akt up-regulation increases resistance to microtubule-directed chemotherapeutic agents through mammalian target of rapamycin. Mol Cancer Ther. 2004; 3: 1605-13.

21. Yang SX, Costantino JP, Kim C, Mamounas EP, Nguyen D, Jeong JH, et al. Akt phosphorylation at Ser473 predicts benefit of paclitaxel chemotherapy in node-positive breast cancer. Journal of clinical oncology : official journal of the American Society of Clinical Oncology. 2010; 28: 2974-81.

22. Hirschey MD, DeBerardinis RJ, Diehl AM, Drew JE, Frezza C, Green MF, et al. Dysregulated metabolism contributes to oncogenesis. Seminars in cancer biology. 2015; 35 Suppl: S129-50.

23. Jones RG, Thompson CB. Tumor suppressors and cell metabolism: a recipe for cancer growth. Genes \& development. 2009; 23: 537-48

24. DeBerardinis RJ, Lum JJ, Hatzivassiliou G, Thompson CB. The biology of cancer: metabolic reprogramming fuels cell growth and proliferation. Cell metabolism. 2008: 7: 11-20.

25. Saraswathy M, Gong S. Recent developments in the co-delivery of siRNA and small molecule anticancer drugs for cancer treatment. Materials Today. 2014; 17: 298-306

26. Miles D vMG, Seidman AD. Combination versus sequential single-agent therapy in metastatic breast cancer. The Oncologist. 2002; 7: 13-9.

27. Atienzar FA, Tilmant K, Gerets HH, Toussaint G, Speeckaert S, Hanon E, et al. The use of real-time cell analyzer technology in drug discovery: defining optimal cell culture conditions and assay reproducibility with different adherent cellular models. Journal of biomolecular screening. 2011; 16: 575-87.

28. Tian $D$, Zhang $W, H e J$, Liu $Y$, Song $Z$, Zhou $Z$, et al Novel, real-time cell analysis for measuring viral cytopathogenesis and the efficacy of neutralizing antibodies to the 2009 influenza A (H1N1) virus. PloS one. 2012; 7: e31965.

29. Urcan E, Haertel U, Styllou M, Hickel R, Scherthan H, Reichl FX. Real-time xCELLigence impedance analysis of the cytotoxicity of dental composite components on human gingival fibroblasts. Dental materials : official publication of the Academy of Dental Materials. 2010; 26: 51-8.

30. Dowling CM, Herranz Ors $C$, Kiely PA. Using real-time impedance-based assays to monitor the effects of fibroblast-derived media on the adhesion, proliferation, migration and invasion of colon cancer cells. Bioscience reports. 2014; 34 .

31. Yuan DD, Zhu ZX, Zhang X, Liu J. Targeted therapy for gastric cancer: Current status and future directions (Review). Oncology reports. 2016; 35: $1245-54$.
32. Swanton C, Marani M, Pardo O, Warne PH, Kelly G, Sahai E, et al. Regulators of mitotic arrest and ceramide metabolism are determinants of sensitivity to paclitaxel and other chemotherapeutic drugs. Cancer cell. 2007; 11: 498-512.

33. Rebucci M, Michiels C. Molecular aspects of cancer cell resistance to chemotherapy. Biochemical pharmacology. 2013; 85: 1219-26.

34. Hennessy BT, Smith DL, Ram PT, Lu Y, Mills GB. Exploiting the PI3K/AKT pathway for cancer drug discovery. Nature reviews Drug discovery. 2005; 4: 988-1004.

35. Fresno Vara JA, Casado E, de Castro J, Cejas P, Belda-Iniesta C, Gonzalez-Baron M. PI3K/Akt signalling pathway and cancer. Cancer treatment reviews. 2004; 30: 193-204

36. Okuzumi T, Fiedler D, Zhang C, Gray DC, Aizenstein B, Hoffman R, et al. Inhibitor hijacking of Akt activation. Nature chemical biology. 2009; 5: 484-93.

37. Zhang H, Su X, Guo L, Zhong L, Li W, Yue Z, et al. Silencing SATB1 inhibits the malignant phenotype and increases sensitivity of human osteosarcoma U2OS cells to arsenic trioxide. International journal of medical sciences. 2014; 11: $1262-9$

38. Zhang L, Lu Z, Zhao Q, Huang J, Shen H, Zhang Z. Enhanced chemotherapy efficacy by sequential delivery of siRNA and anticancer drugs using PEI-grafted graphene oxide. Small. 2011; 7: 460-4.

39. Cheng JC, Sakamoto KM. The emerging role of RNA interference in the design of novel therapeutics in oncology. Cell Cycle. 2004; 3: 1398-401.

40. Scott CW, Peters MF. Label-free whole-cell assays: expanding the scope of GPCR screening. Drug discovery today. 2010; 15: 704-16.

41. Franke TF, Hornik CP, Segev L, Shostak GA, Sugimoto C. PI3K/Akt and apoptosis: size matters. Oncogene. 2003; 22: 8983-98.

42. Das TP, Suman S, Alatassi H, Ankem MK, Damodaran C. Inhibition of AKT promotes FOXO3a-dependent apoptosis in prostate cancer. Cell death \& disease. 2016; 7: e2111.

43. Garofalo M, Quintavalle C, Zanca C, De Rienzo A, Romano G, Acunzo M, et al. Akt regulates drug-induced cell death through Bcl-w downregulation. PloS one. 2008; 3: e4070.

44. Jing LIU AL. Role of JNK activation in apoptosis; A double-edged sword. Cell Research. 2005; 15: 36-42. 